Journal for .... ImmunoTherapy of Cancer

\section{Adjuvant nivolumab for stage III/IV melanoma: evaluation of safety outcomes and association with recurrence-free survival}

To cite: Mandalá M, Larkin J, Ascierto PA, et al. Adjuvant nivolumab for stage III/IV melanoma: evaluation of safety outcomes and association with recurrence-free survival. Journal for ImmunoTherapy of Cancer 2021;9:e003188. doi:10.1136/ jitc-2021-003188

- Additional supplemental material is published online only. To view, please visit the journal online (http://dx.doi.org/10. 1136/jitc-2021-003188).

Accepted 09 August 2021
Check for updates

(C) Author(s) (or their employer(s)) 2021. Re-use permitted under CC BY-NC. No commercial re-use. See rights and permissions. Published by BMJ.

For numbered affiliations see end of article.

Correspondence to Dr Jeffrey Weber; Jeffrey.Weber@nyulangone.org

\section{ABSTRACT}

Background Several therapeutic options are now available in the adjuvant melanoma setting, mandating an understanding of their benefit-risk profiles in order to make informed treatment decisions. Herein we characterize adjuvant nivolumab select (immune-related) treatment-related adverse events (TRAEs) and evaluate possible associations between safety and recurrence-free survival (RFS) in the phase III CheckMate 238 trial.

Methods Patients with resected stage IIIB-C or IV melanoma received nivolumab $3 \mathrm{mg} / \mathrm{kg}$ every 2 weeks $(\mathrm{n}=452)$ or ipilimumab $10 \mathrm{mg} / \mathrm{kg}$ every 3 weeks for four doses and then every 12 weeks $(n=453)$ for up to 1 year or until disease recurrence, unacceptable toxicity, or consent withdrawal. First-occurrence and all-occurrence select TRAEs were analyzed within discrete time intervals: from 0 to 3 months of treatment, from $>3-12$ months of treatment, and from the last dose (regardless of early or per-protocol treatment discontinuation) to 100 days after the last dose. Possible associations between select TRAEs and RFS were investigated post randomization in 3-month landmark analyses and in Cox model analyses (including a time-varying covariate of select TRAE), within and between treatment groups.

Results From the first nivolumab dose to 100 days after the last dose, first-occurrence select TRAEs were reported in $67.7 \%$ (306/452) of patients. First-occurrence select TRAEs were reported most frequently from 0 to 3 months (48.0\%), during which the most common were pruritus (15.5\%) and diarrhea (15.3\%). Most select TRAEs resolved within 6 months. There was no clear association between the occurrence (or not) of select TRAEs and RFS by landmark analysis or by Cox model analysis within treatment arms or comparing nivolumab to the ipilimumab comparator arm. Conclusion Results of this safety analysis of nivolumab in adjuvant melanoma were consistent with its established safety profile. In the discrete time intervals evaluated, most first-occurrence TRAEs occurred early during treatment and resolved. No association between RFS and select TRAEs was evident.
Trial registration number NCT02388906.

\section{INTRODUCTION}

There is a need for effective adjuvant treatment for patients with high-risk, stage III/ IV resected melanoma given that surgical resection alone, while critical for the treatment of the primary tumor, is associated with high rates of relapse. Historically, 5-year recurrence rates in high-risk stage III melanoma after surgery were $50 \%-80 \%^{1-4}$ and the 5-year overall survival (OS) rate was approximately $60 \% .^{5} 6$ Adjuvant systemic treatment of melanoma was initially limited to interferon- $\alpha$ and ipilimumab (which is only approved in the USA). ${ }^{78}$ However, new therapies have recently become available, such as the anti-programmed death 1 (PD-1) agents nivolumab and pembrolizumab, and the BRAF/MEK kinase inhibitor combination of dabrafenib plus trametinib. ${ }^{910}$ In the phase III CheckMate 238 trial (ClinicalTrials. gov identifier: NCT02388906), nivolumab $3 \mathrm{mg} / \mathrm{kg}$ demonstrated significantly longer recurrence-free survival (RFS) and a lower rate of grade $3-4$ adverse events (AEs) than ipilimumab at $10 \mathrm{mg} / \mathrm{kg}$ in patients with completely resected stage IIIB/IIIC or stage IV melanoma. ${ }^{11}$ Based on these results, adjuvant nivolumab was approved globally. With extended follow-up, the RFS benefit with nivolumab was sustained compared with ipilimumab (estimated 48-month RFS rates, $51.7 \%$ for nivolumab vs $41.2 \%$ for ipilimumab; HR: 0.71 ; $95 \%$ CI: 0.60 to 0.86 ; $\mathrm{p}=0.0003)$. $^{12}$ 
With several therapeutic options now available in the adjuvant melanoma setting, an understanding of the benefit-risk profile of each is needed to make informed treatment decisions. Here we provide a comprehensive evaluation of nivolumab safety in the adjuvant melanoma setting and evaluate possible associations with efficacy using data from the CheckMate 238 trial.

\section{PATIENTS AND METHODS \\ Patients}

Eligible patients were age 15 years or older and had stage IIIB/IIIC or stage IV melanoma (per the 2009 classification of the American Joint Committee on Cancer (AJCC), seventh edition, ${ }^{13}$ an Eastern Cooperative Oncology Group performance status of 0 or 1 , and histologically confirmed melanoma with metastases to regional lymph nodes or distant metastases that had been surgically resected. Patients were required to have had complete resection of disease within the 12 weeks before randomization. Patients with resected brain metastases were eligible to participate. Exclusion criteria included a diagnosis of ocular or uveal melanoma, active autoimmune disease, previous non-melanoma cancer without complete remission for more than 3 years, systemic glucocorticoid use, and previous systemic treatment for melanoma (prior interferon therapy was permitted).

\section{Study design}

CheckMate 238 is an ongoing phase III, randomized, double-blind trial (online supplemental appendix figure A1). ${ }^{11} 12$ Patients were enrolled from March 30, 2015, to November 30, 2015, at 130 centers in 25 countries. Patients were assigned (1:1) to receive either nivolumab $3 \mathrm{mg} / \mathrm{kg}$ every 2 weeks or ipilimumab $10 \mathrm{mg} / \mathrm{kg}$ every 3 weeks for four doses and then every 12 weeks, each for a maximum of 1 year or until disease recurrence, unacceptable toxicity, or withdrawal of consent when these occur before 1 year.

\section{Trial endpoints and assessments}

The primary endpoint was RFS assessed in the intentionto-treat population; secondary endpoints included OS and safety. ${ }^{11}{ }^{12}$ Patients were assessed radiologically and clinically for recurrence every 12 weeks for the first 2 years after randomization and every 6 months thereafter for up to 5 years. RFS was defined as the time from randomization until the date of the first recurrence (local, regional, or distant metastasis), new primary melanoma, or death from any cause. AE data were collected according to Common Terminology Criteria for Adverse Events, version 4.0. ${ }^{14}$ Patients were followed for safety for up to 100 days following their last dose and select (ie, immune-related) treatment-related adverse events (TRAEs) are investigated here. Select TRAEs were determined based on a prespecified list of terms from the Medical Dictionary for Regulatory Activities. Select TRAEs are those with presumed immune-related etiology that may be mitigated by early recognition and potential management with immunosuppression and for which multiple event terms may be used to describe a single type. As in previous nivolumab reports, those included here were endocrine, gastrointestinal, hepatic, pulmonary, renal, and skin. Endpoints that involve corticosteroid use are reported for both corticosteroid use (without other immunosuppressants) and for corticosteroid use including other immunosuppressants.

Since the approval of PD-1 inhibitors, ipilimumab is no longer a standard of care for the adjuvant treatment of melanoma and therefore, nivolumab treatment is the main focus of this study. However, coordinating data for ipilimumab are included in the online supplemental appendix.

\section{Safety analysis}

The initial 18-month minimum follow-up analysis for this trial has been previously reported and included a final safety analysis based on data up to 30 days after the last dose. ${ }^{112}$ This report presents the full safety analysis for the entire period for which safety data were collected (100 days after the last dose, per protocol). Select TRAE data collected during treatment and for up to 100 days from the last dose were evaluated within the following discrete time intervals: $0-3$ months of treatment, $>3-12$ months of treatment, and from the last dose (regardless of early or per-protocol treatment discontinuation) to 100 days after the last dose. First-occurrence and all-occurrence (including repeat occurrences) select TRAEs were evaluated within these time intervals. Overall time to onset and time to resolution of select TRAEs were analyzed for events reported between first dose and 100 days after last dose of study therapy.

\section{Association between select TRAEs and RFS}

A possible association between select TRAEs and RFS was investigated using efficacy data with a minimum of 24 months of follow-up. All RFS associations were estimated using Kaplan-Meier landmark analysis accounting for guarantee-time bias ${ }^{15}$ within the first 3 months after randomization. Comparisons were made between patients with and without a select TRAE in the first 3 months, the median time to onset for most select TRAEs. In addition, a Cox model was used to evaluate the association between the occurrence of select TRAEs (or not) and RFS, both within treatment groups and comparing nivolumab to ipilimumab. The model included a time-varying covariate with a value of 0 before the $\mathrm{AE}$ onset and 1 after onset and was adjusted for AJCC seventh edition disease stage (stage IIIB, IIIC, IV, or other), sex, and age $(<65$ years or $\geq 65$ years). The model included the treatment indicator and the product of the treatment indicator and the time-varying select TRAE category indicator. HRs (and 95\% CIs) were estimated for the randomized treatment in the presence and absence of select TRAEs. The $p$ values provided are descriptive. 
RESULTS

\section{Patients}

A total of 906 patients were randomized to either nivolumab $(\mathrm{n}=453)$ or ipilimumab $(\mathrm{n}=453)$. Patient characteristics were reported previously (online supplemental appendix table A1). ${ }^{11}$ One patient who was randomized to the nivolumab group did not receive treatment. Patients received a median of 24 doses (range: 1-26) of nivolumab and 4 doses (range: $1-7$ ) of ipilimumab. One year of treatment was completed by $61 \%$ of nivolumabtreated patients and $27 \%$ of ipilimumab-treated patients, with the majority of treatment discontinuations due to disease recurrence with nivolumab (121/177) and to study drug toxicity with ipilimumab $(208 / 331){ }^{11}$

\section{Safety analysis}

In the nivolumab group, first-occurrence, any-grade select TRAEs from the first dose to 100 days after the last dose were reported in 306 of 452 patients (67.7\%; grade 1-2: $\mathrm{n}=285(63.1 \%)$; grade 3-4: $\mathrm{n}=21$ (4.6\%); online supplemental appendix table A2). Among all first-occurrence select TRAEs with nivolumab, the most common were diarrhea $(24.8 \%)$, pruritus $(23.5 \%)$ and rash $(20.6 \%)$. First-occurrence any-grade endocrine select TRAEs occurring in $\geq 1 \%$ of nivolumab-treated patients included hypothyroidism $(11.3 \%)$, hyperthyroidism $(8.0 \%)$, thyroiditis
(2.0\%), hypophysitis $(1.8 \%)$, and adrenal insufficiency $(1.3 \%)$. Although not reported as immune related, first-occurrence any-grade pneumonitis and diabetes mellitus occurred in $1.3 \%$ and $0.4 \%$ of nivolumab-treated patients, respectively, and first-occurrence any-grade paresthesia, peripheral neuropathy, and axonal neuropathy (neurologic TRAEs of special interest) were reported in $2.7 \%, 0.4 \%$, and $0.2 \%$ of nivolumab-treated patients, respectively.

First-occurrence select TRAEs with nivolumab were reported in $48.0 \%$ of patients from 0 to 3 months of treatment (online supplemental appendix table A3), 35.0\% of patients from $>3$ to 12 months of treatment (online supplemental appendix table A4), and $11.9 \%$ of patients from the last dose to 100 days after the last dose (online supplemental appendix table A5). First-occurrence select TRAEs reported in at least $2 \%$ of nivolumab-treated patients during any of the three time intervals evaluated are depicted in figure 1 . During the time period from 0 to 3 months of treatment, the most common of these firstoccurrence select TRAEs were pruritus at $15.5 \%$, diarrhea at $15.3 \%$, rash at $11.7 \%$ and hyperthyroidism at $7.1 \%$ (figure 1A; online supplemental appendix table A3). During the time period from $>3$ to 12 months on treatment, the most common first-occurrence select TRAEs
A On treatment (any-grade, $\geq 2 \%$ )

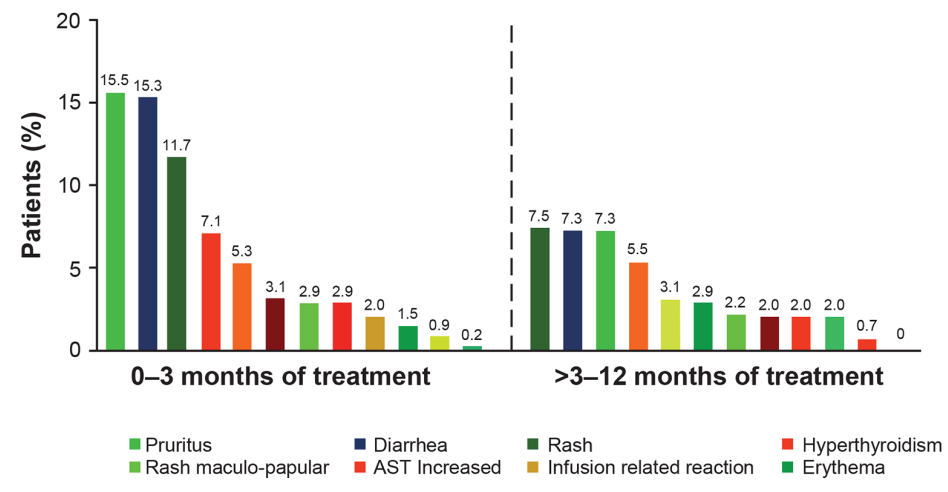

B From last dose (any-grade, $\geq 2 \%$ )

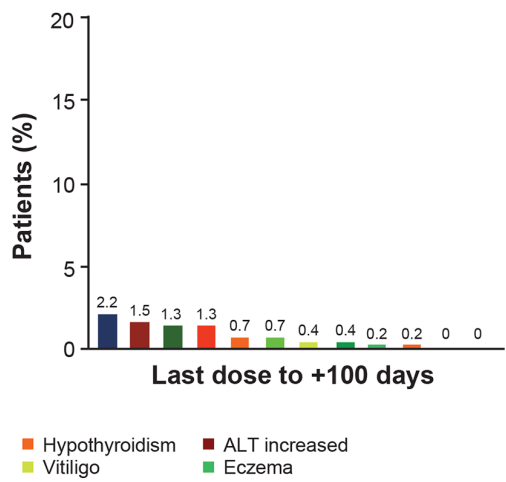

D From last dose (grade 3-4)

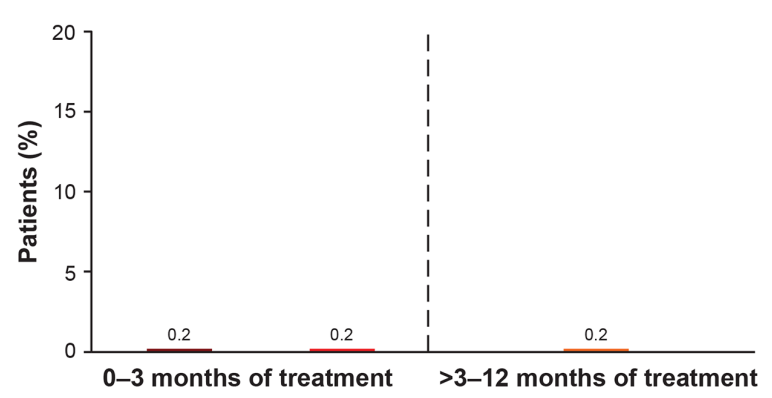

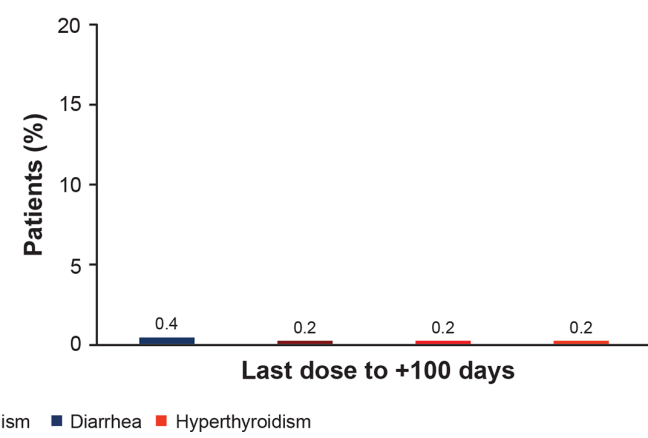

Figure 1 Frequencies of first-occurrence select treatment-related adverse events reported in $\geq 2 \%$ of nivolumab-treated patients during any time interval. (A) Any-grade occurring on treatment. (B) Any-grade events occurring from the last dose to 100 days after the last dose. (C) Grade 3-4 events, of those shown in panel A. (D) Grade 3-4 events, of those shown in panel B. TRAEs were coded using the Medical Dictionary for Regulatory Activities, V.20.0, and preferred terms may not be mutually exclusive. ALT; alanine aminotransferase. AST; aspartate aminotransferase. 
were rash at $7.5 \%$, diarrhea at $7.3 \%$, pruritus at $7.3 \%$, and hypothyroidism at $5.3 \%$ (figure $1 \mathrm{~A}$; online supplemental appendix table A4), and from the last dose to 100 days after last dose, the most common was diarrhea at $2.2 \%$ (figure 1B; online supplemental appendix table A5).

Similar safety analyses for the three time periods were also performed to evaluate the incidence of alloccurrence select TRAEs (including repeat occurrences), as well as with first occurrence and all-occurrence allinclusive TRAEs, with similar results obtained (data not shown). In nivolumab-treated patients, $39.7 \%$ of alloccurrence, all-inclusive TRAEs (1079/2716) occurred from 0 to 3 months of treatment, $46.1 \%(1252 / 2716)$ occurred from $>3$ to 12 months of treatment, and $14.2 \%$ $(385 / 2716)$ occurred from the last dose to 100 days after the last dose. Of the all-inclusive TRAEs with nivolumab, $39.9 \%(1084 / 2716)$ were considered select TRAEs and of those, $40.4 \%(438 / 1084)$ occurred from 0 to 3 months of treatment, $45.8 \%(496 / 1084)$ from after 3 months to 12 months of treatment, and $13.8 \%(150 / 1084)$ from the last dose to 100 days after the last dose. Discontinuation due to all-inclusive TRAEs and select TRAEs occurred in $7.7 \%(35 / 452)$ and $5.1 \%(23 / 452)$ of patients, respectively. All-occurrence endocrine TRAEs from first dose to 100 days following last dose are presented in online supplemental appendix table A6.
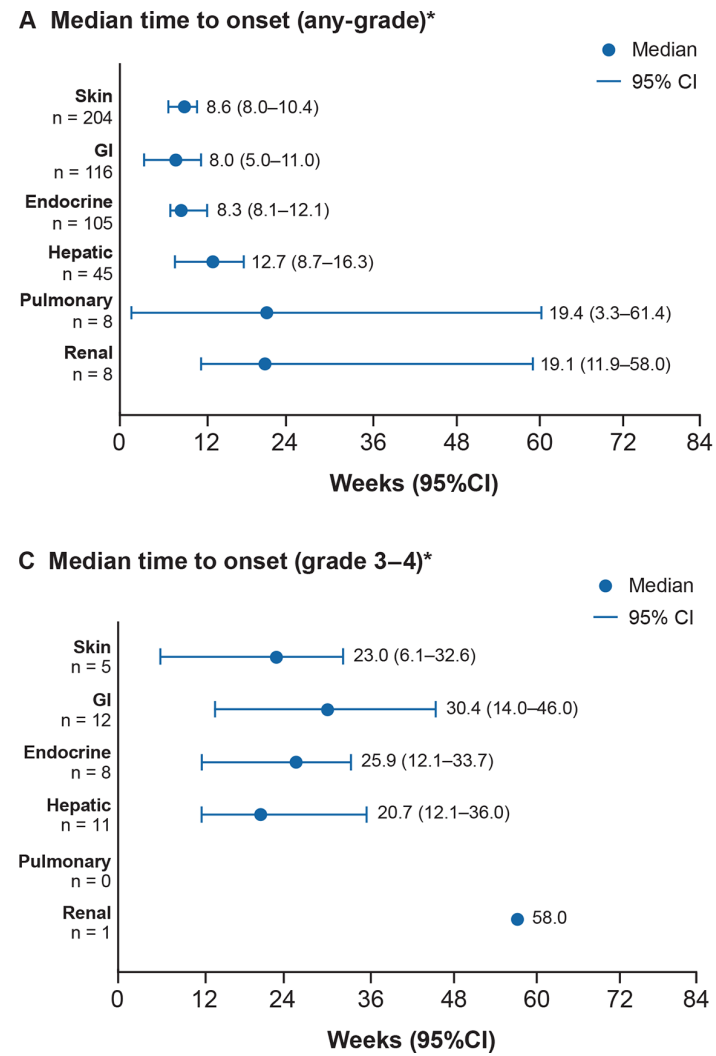

Figure 2 Time to onset (A) and time to resolution (B) of any grade select treatment-related adverse events from first dose of nivolumab to 100 days after the last dose; time to onset (C) and time to resolution (D) of grade 3-4 select treatment-related adverse events from first dose of nivolumab to 100 days after the last dose. *Numbers of patients with onset of an adverse event. †Numbers of patients whose adverse event resolved out of the numbers of patients with onset of an adverse event. GI, gastrointestinal; NR, not reached.
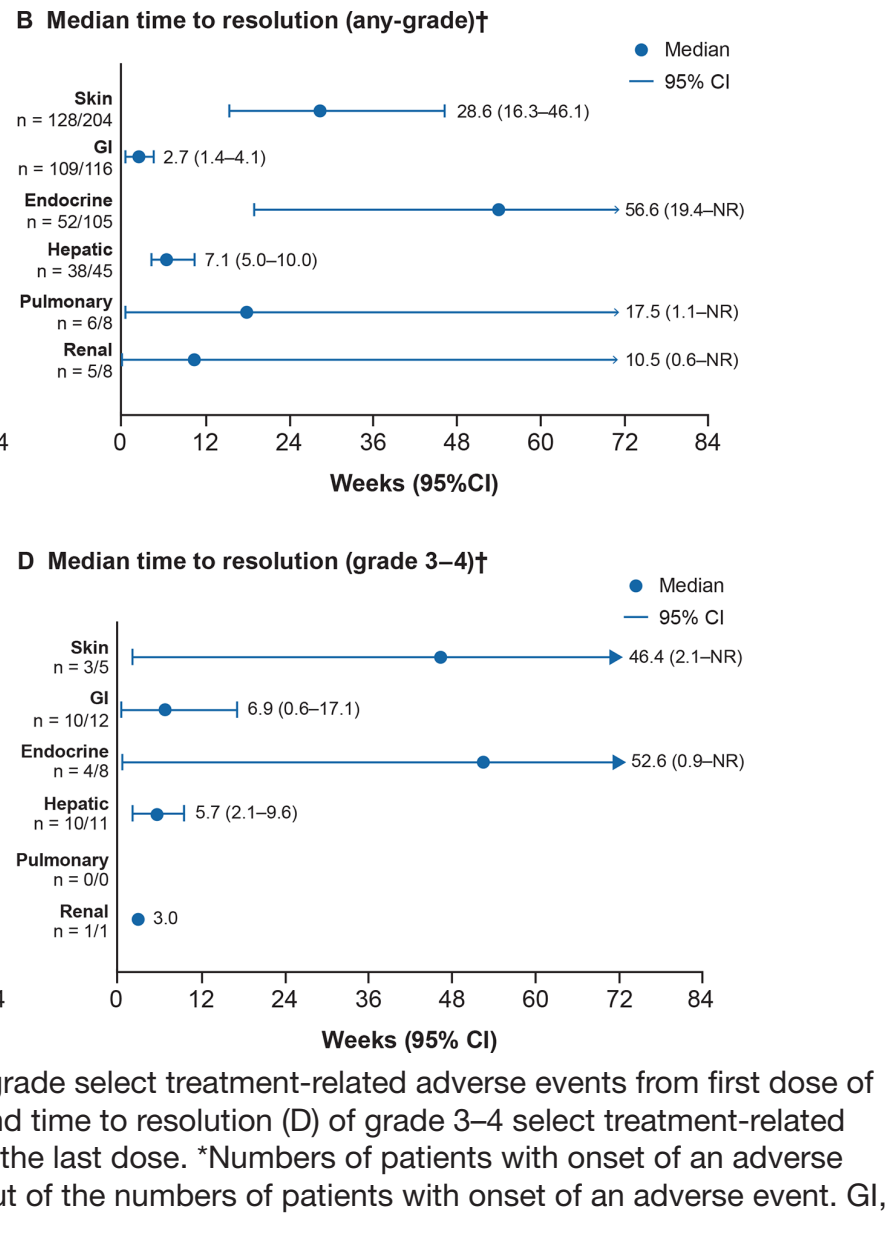

Median time to onset of select TRAEs with nivolumab was approximately 8 weeks for skin, gastrointestinal, and endocrine AEs; 13 weeks for hepatic AEs; and 19 weeks for pulmonary and renal AEs (figure 2A). Most select TRAEs with nivolumab resolved within 6 months (figure 2B), although the median time to resolution of endocrine TRAEs was more than 1 year. Endocrine AEs were not considered resolved in $50.5 \%(53 / 105)$ of nivolumabtreated patients.

Corticosteroids (and not other immunosuppressant agents) were administered to $31.0 \%$ (140/452) of nivolumab-treated patients for the management of select TRAEs. The median duration (range) of corticosteroid use was 1.8 months $(0-18.0)$ and corticosteroids or other immunosuppressant agents were administered to $45.4 \%$ $(205 / 452)$ of nivolumab-treated patients. The median duration (range) of use of corticosteroids or other immunosuppressant agents was 3.7 months $(0-21.3)$.

\section{Safety and efficacy association}

In Kaplan-Meier 3-month landmark analyses, there was no clear association between the occurrence of a select TRAE in the first 3 months of nivolumab treatment and RFS (figure 3). In similar landmark analyses evaluating RFS in patients with and without select TRAEs, no clear association was observed between RFS and select skin 


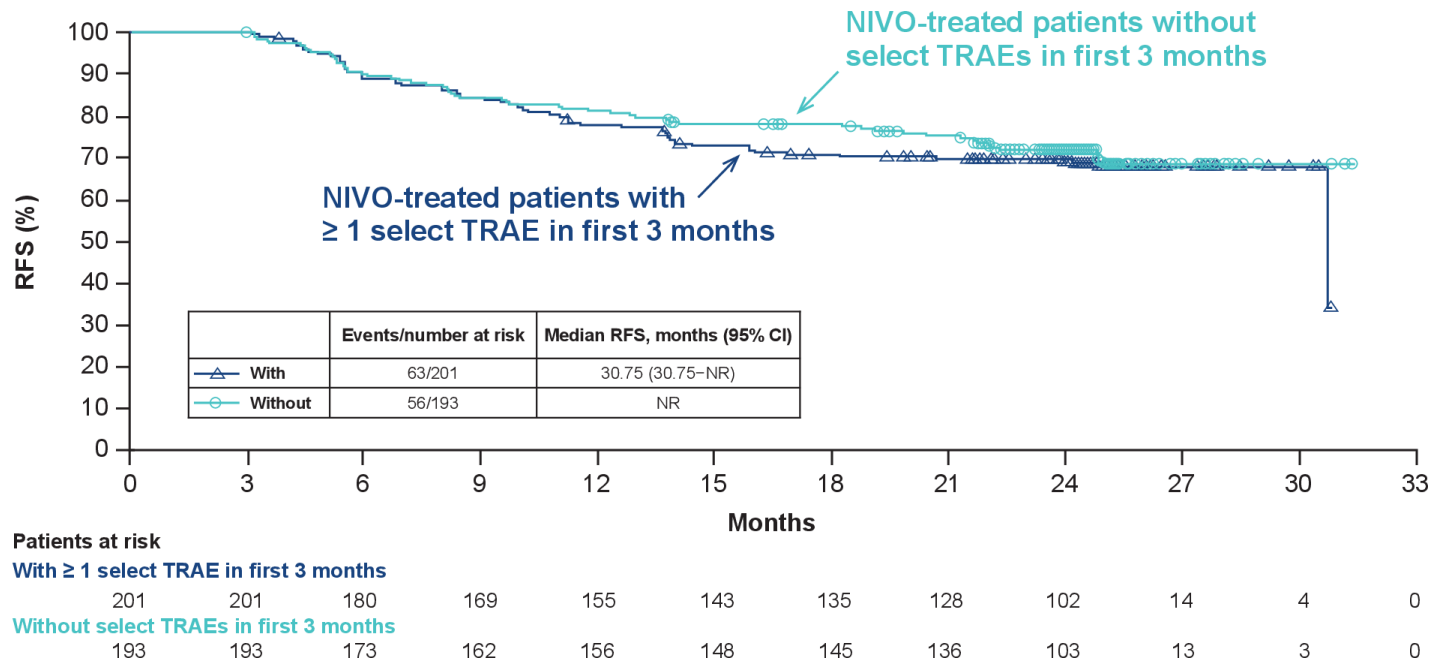

Figure 3 Three-month landmark analysis of RFS in nivolumab-treated patients with and without early select TRAEs. NIVO, nivolumab; NR, not reached; RFS, recurrence-free survival; TRAE, treatment-related adverse event.

TRAEs, select gastrointestinal TRAEs, the use of corticosteroids as the sole type of immunosuppressant agent, or the use of immunosuppressants such as corticosteroids or other agents (online supplemental appendix figure A2). Patients treated with nivolumab and with corticosteroid use had a slight numerical decrease in RFS versus those without corticosteroid use, but because of the very small number of patients available in the former group, interpretation of these data is very limited. Results of additional landmark analyses of clinical interest (ie, those evaluating the association between RFS and the occurrence of vitiligo, discontinuation of study treatment for any reason, or discontinuation of study treatment due to study drug toxicity) were inconclusive because one or both of the treatment subgroups contained 10 or fewer patients (data not shown). In a cox model analysis, the occurrence of a select TRAE reported between first study dose and 100 days after last study dose was not associated with RFS in patients treated with nivolumab (table 1). HR for RFS in patients without a select TRAE compared with patients with a select TRAE was 0.97 (95\% CI: 0.70 to $1.34 ; \mathrm{p}=0.858)$, with similar results in skin and gastrointestinal select TRAEs, and select TRAEs with or without the use of corticosteroids or immune suppressants use. In addition, compared with the ipilimumab arm, the reduction in the hazard of recurrence or death was not significant $(p=0.1400)$ in patients treated with nivolumab with or without the onset of select TRAEs (table 2).

Coordinating analyses were performed for ipilimumab. As expected, treatment with ipilimumab led to greater toxicity than nivolumab (online supplemental appendix figure A3, tables A7-A11). In ipilimumab-treated patients, median time to both onset of select TRAEs and resolution of most select TRAEs was less than 12 weeks (online supplemental appendix figure A4). There was no clear association between RFS and a select TRAE occurring in the first 3 months of ipilimumab treatment (online supplemental appendix figure A5), or with the various other select TRAE categories analyzed (online supplemental appendix figure A6). Moreover, as with nivolumab treatment, the presence or absence of select TRAEs reported between first study dose and 100 days after last study dose (time-varying covariate) was not associated with RFS in the ipilimumab group (HR: $0.62 ; 95 \% \mathrm{CI}: 0.41$ to 0.96 ; $\mathrm{p}=0.0301$ (to account for multiple comparisons, $\mathrm{p} \leq 0.01$

Table 1 Nivolumab treatment effect in the absence compared with the presence of select treatment-related adverse events

\begin{tabular}{llc}
\hline Select TRAE type & Nivolumab RFS HR (95\% Cl)* & P value† \\
\hline Any select TRAE & $0.97(0.70$ to 1.34) & 0.8583 \\
Skin select TRAEs & $1.27(0.91$ to 1.76) & 0.1577 \\
Gastrointestinal select TRAEs & $0.89(0.62$ to 1.28) & 0.5355 \\
Select TRAEs with corticosteroid or immunosuppressant use & $0.76(0.52$ to 1.09) & 0.1319 \\
Select TRAEs without corticosteroid or immunosuppressant use & $1.06(0.77$ to 1.46) & 0.7063 \\
\hline
\end{tabular}

${ }^{*}$ Cox model was used which included a time-varying indicator for select TRAEs; Cox model was adjusted for AJCC-7 stage provided at randomization, sex, and age; HR is absence over presence of TRAEs.

†P value is calculated for the time-varying indicator for select TRAEs. Due to multiple hypothesis testing, the Bonferroni-adjusted significant $p$ value for each test is 0.01 .

RFS, recurrence-free survival; TRAE, treatment-related adverse event. 
Table 2 Treatment effect of nivolumab compared with ipilimumab in the presence and absence of select TRAEs

\begin{tabular}{|c|c|c|}
\hline Select TRAE status and treatment arm & RFS HR $(95 \% \mathrm{Cl})^{*}$ & P valuet \\
\hline \multicolumn{3}{|l|}{ Select TRAE } \\
\hline Ipilimumab & 1 & \\
\hline Without/before select TRAE & 0.96 (0.61 to 1.51$)$ & 0.1400 \\
\hline After select TRAE & 0.65 (0.51 to 0.83$)$ & \\
\hline \multicolumn{3}{|l|}{ Skin select TRAEs } \\
\hline Ipilimumab & 1 & \\
\hline Without/before skin select TRAEs & 0.81 (0.62 to 1.08$)$ & 0.0716 \\
\hline After skin select TRAEs & 0.55 (0.41 to 0.76$)$ & \\
\hline \multicolumn{3}{|l|}{ Gastrointestinal select TRAEs } \\
\hline Ipilimumab & 1 & \\
\hline Without/before gastrointestinal select TRAEs & $0.62(0.49$ to 0.80$)$ & 0.2451 \\
\hline After gastrointestinal select TRAEs & 0.81 (0.56 to 1.19$)$ & \\
\hline \multicolumn{3}{|l|}{ Select TRAEs with corticosteroid or immunosuppressant use } \\
\hline Ipilimumab & 1 & \\
\hline Without/before select TRAEs with corticosteroid or immunosuppressant use & $0.62(0.47$ to 0.82$)$ & 0.1412 \\
\hline After select TRAEs with corticosteroid or immunosuppressant use & $0.87(0.61$ to 1.24$)$ & \\
\hline \multicolumn{3}{|l|}{ Select TRAEs without corticosteroid or immunosuppressant use } \\
\hline Ipilimumab & 1 & \\
\hline Without/before select TRAEs without corticosteroid or immunosuppressant use & 0.96 (0.67 to 1.39$)$ & 0.0392 \\
\hline After select TRAEs without corticosteroid or immunosuppressant use & $0.60(0.47$ to 0.78$)$ & \\
\hline
\end{tabular}

${ }^{*}$ Cox model was used which included treatment indicator, a time-varying indicator for select TRAEs and the interaction term between the variables; Cox model was adjusted for AJCC-7 stage provided at randomization, sex, and age; HR is nivolumab over ipilimumab. †P value is calculated for the test of an HR difference in the presence and absence of TRAEs (ie, the difference between the 2 HRs). Due to multiple hypothesis testing, the Bonferroni-adjusted significant $p$ value for each test is 0.01 .

HR, hazard ratio; RFS, recurrence-free survival; TRAE, treatment-related adverse event.

is considered significant, although, descriptive); online supplemental appendix table A12).

\section{DISCUSSION}

Results of this safety analysis of adjuvant nivolumab or ipilimumab in patients with resected stage IIIB/IIIC or stage IV melanoma in the CheckMate 238 trial were consistent with the established safety profiles of these agents in more advanced settings. This analysis revealed that most first-occurrence select TRAEs with nivolumab took place early during treatment and that an association between the development of early TRAEs with nivolumab and RFS was not evident when the data were analyzed by two different statistical techniques.

Select TRAEs with adjuvant nivolumab and the timing of their first occurrence were consistent with the established safety profile of nivolumab monotherapy. ${ }^{16}$ Overall, $67.7 \%$ of patients had a first-occurrence select TRAE. In the discrete time periods analyzed here, $48.0 \%$ of patients reported a first-occurrence select TRAEs within the first 3 months of treatment. During this period, the most common first-occurrence, select TRAEs with nivolumab were pruritus $(15.5 \%)$, diarrhea $(15.3 \%)$, rash $(11.7 \%)$, and hyperthyroidism $(7.1 \%)$. These results were generally consistent with those of a pooled analysis of nivolumab in patients with advanced melanoma from four studies, in which the most common any-grade all-inclusive TRAEs were fatigue $(24.8 \%)$, pruritus $(17.2 \%)$, diarrhea $(12.7 \%)$, and rash $(12.7 \%) .{ }^{16}$ Considering total events, approximately $40 \%$ of all-occurrence (including repeat occurrences) all-inclusive TRAEs and of all-occurrence select TRAEs were reported within the first 3 months of treatment.

As a class, immune checkpoint inhibitors are associated with select (ie, immune-related) AEs. ${ }^{9}{ }^{17}$ In this analysis, most select TRAEs with nivolumab occurred within approximately 3 months of treatment initiation and generally resolved within 6 months of occurrence. However, the median time to resolution of endocrine select TRAEs was more than 1 year, likely because some (eg, thyroid-related AEs) — even though medically controlled-may not have been considered resolved (even if asymptomatic) if patients continued to require medical treatment (eg, hormone replacement therapy). These results confirmed that select TRAEs with adjuvant nivolumab are manageable using established safety guidelines, as demonstrated in the advanced/metastatic melanoma setting. ${ }^{17}$ 
Results of some analyses have suggested an association between TRAEs and response to immune checkpoint inhibitors in patients with advanced or metastatic melanoma, although the evidence has been contradictory. ${ }^{16-21}$ In the pooled safety analysis with nivolumab for metastatic melanoma, objective response rate (ORR) was significantly higher in patients who experienced select TRAEs than in those who did not, but no association was noted between occurrence of select TRAEs and progression-free survival using a landmark approach. ${ }^{16}$ In a pooled analysis of two phase I studies in patients with advanced melanoma treated with nivolumab, OS was significantly longer in patients who experienced rash or vitiligo than in those who did not in a 12-week landmark analysis, although an association was not observed for the other select TRAEs evaluated. ${ }^{18}$ In an observational study in patients with metastatic melanoma treated with pembrolizumab, ORR was significantly higher in patients with vitiligo than in those without this $\mathrm{AE}$, but there was no association between vitiligo and OS in landmark analyses. ${ }^{19}$ In an expanded access program with pembrolizumab in advanced melanoma, there was no significant difference in ORR between patients who experienced select TRAEs and those who did not. ${ }^{20}$ Also in advanced melanoma, disease control and OS was not statistically associated with immune-related AEs in patients treated with ipilimumab $10 \mathrm{mg} / \mathrm{kg}$ in an analysis of three phase II trials. ${ }^{21}$ These contradictory findings underscore the need for more extensive research to identify novel biological markers to predict checkpoint inhibitor-related toxicity and response to therapy in patients with melanoma presenting with early or late-stage disease.

The results of the Kaplan-Meier 3-month landmark analyses of CheckMate 238 suggested that there was no clear association between RFS and select TRAEs occurring in the first 3 months of nivolumab or ipilimumab treatment. These results contrast with those of a timevarying covariate analysis from the KEYNOTE-054 trial in patients with high-risk stage III melanoma treated with adjuvant pembrolizumab or placebo. ${ }^{22}$ In that analysis, the occurrence of an any-grade select TRAE with pembrolizumab was associated with a longer RFS (HR: $0.61 ; 95 \%$ CI: 0.39 to $0.95 ; \mathrm{p}=0.03) .{ }^{22}$ In contrast, a similar analysis conducted here with CheckMate 238 data found no relationship for nivolumab treatment between the occurrence of select TRAEs at any time and RFS (HR: 0.97; $95 \%$ CI: 0.70 to 1.34 ; $\mathrm{p}=0.8583$ ). Although CheckMate 238 and KEYNOTE-054 enrolled largely overlapping patient populations, the different outcomes noted between the two post-hoc analyses cannot be easily explained and may be partially related to unaccounted inherent differences in baseline patient characteristics. Lastly, the list of specific AEs included in the select TRAEs evaluated in CheckMate 238 differed slightly from the immune-related AEs evaluated in KEYNOTE-054, which included endocrine AEs, pneumonitis/interstitial lung disease, sarcoidosis, vitiligo, severe skin reaction, colitis, pancreatitis, hepatitis, nephritis, uveitis, myositis, or myocarditis. ${ }^{22}$ The potentially different definition used in CheckMate 238 may have partially contributed to the difference noted in the results.

Findings from this safety analysis of adjuvant nivolumab and ipilimumab in patients with resected stage IIIB-C or stage IV melanoma from the CheckMate 238 trial are consistent with the established safety profile of each agent. In the discrete time intervals evaluated, $40 \%$ of first-occurrence select TRAEs with nivolumab occurred within the first 3 months of treatment. Most select TRAEs with nivolumab resolved within 6 months of onset. There was no association between treatment-related select AEs and RFS, suggesting that the development of such immune-mediated events may not be predictive of efficacy. Limitations of this analysis included the retrospective nature and the lack of powering for these retrospective landmark and time-varying Cox model analyses. Specifically for the Kaplan-Meier analyses, adjustments required to reduce lead-time bias necessarily resulted in the removal of some patients. Overall, these findings add to the understanding of the benefit-risk profile of adjuvant nivolumab in patients with resected high-risk melanoma and may ultimately help in guiding clinicians and patients to recognize and monitor select TRAEs in this setting.

\section{Author affiliations}

${ }^{1}$ Unit of Medical Oncology, University of Perugia, Perugia, Italy

${ }^{2}$ Royal Marsden NHS Foundation Trust, London, UK

${ }^{3}$ Istituto Nazionale Tumori IRCCS Fondazione Pascale, Napoli, Italy

${ }^{4}$ Unit of Melanoma Medical Oncology, Fondazione IRCCS Istituto Nazionale dei Tumori, Milano, Italy

${ }^{5}$ First Department of Medicine, National and Kapodistrian University of Athens School of Medicine, Athens, Greece

${ }^{6}$ Texas Oncology Baylor Charles A Sammons Cancer Center, Dallas, Texas, USA ${ }^{7}$ Hospital Clínic de Barcelona, Barcelona, Spain

${ }^{8}$ Hospices Civils de Lyon, Pierre Bénite, France

${ }^{9}$ Oncology Center Sf Nectarie Ltd, Craiova, Romania

${ }^{10}$ Aix-Marseille University, APHM Hospital, Marseille, France

${ }^{11}$ Veneto Institute of Oncology IOV-IRCCS, Padua, Italy

${ }^{12}$ General University Hospital Gregorio Marañón, CIBERONC, Madrid, Spain

${ }^{13}$ Princess Margaret Hospital Cancer Centre, Toronto, Ontario, Canada

${ }^{14}$ Center for Immuno-Oncology, University Hospital of Siena, Siena, Italy

${ }^{15}$ Oncology, Mount Sinai Medical Center, Miami Beach, Florida, USA

${ }^{16}$ Clinical Oncology Department, Hospital Universitario Virgen Macarena, Sevilla, Spain

${ }^{17}$ Gallipoli Medical Research Foundation and University of Queensland, Brisbane, Queensland, Australia

${ }^{18}$ University Hospital Královské, Vinohrady, Czech Republic

${ }^{19}$ Tasman Oncology Research Ltd, Southport, Queensland, Australia

${ }^{20}$ Rogel Cancer Center, University of Michigan, Ann Arbor, Michigan, USA

${ }^{21} \mathrm{Sir}$ Charles Gairdner Hospital, University of Western Australia, Nedlands, Western Australia, Australia

${ }^{22}$ Department of Cutaneous Oncology, Moffitt Cancer Center, Tampa, Florida, USA

${ }^{23}$ Bristol Myers Squibb, Princeton, New Jersey, USA

${ }^{24}$ Perlmutter Cancer Center, NYU Langone Health, New York, New York, USA

Correction notice This paper has been updated to amend author details.

Twitter Paolo A Ascierto @PAscierto

Acknowledgements We thank the patients and families who made the study possible and the clinical study teams who participated. In addition, we thank Agnes Balogh and Margarita Askelson for statistical assistance. We also acknowledge Ono Pharmaceutical Company Ltd (Osaka, Japan) for contributions to nivolumab 
development and Dako, an Agilent Technologies, Inc company, for collaborative development of the PD-L1 IHC 28-8 pharmDx assay. This analysis was supported by Bristol Myers Squibb. All authors contributed to and approved the manuscript; writing and editorial assistance was provided by Mark Palangio, Melissa Kirk, and Michele Salernitano at Ashfield Healthcare Communications, funded by Bristol Myers Squibb.

Contributors All authors were involved in conception and design, administrative support, provision of study material or patients, collection and assembly of data, data analysis and interpretation, and manuscript writing. All authors gave final approval of the manuscript to be published and were accountable for all aspects of the work.

Funding CheckMate 238 is supported by Bristol Myers Squibb and Ono Pharmaceutical.

Competing interests MM: Consulting or Advisory Role: Bristol Myers Squibb, Novartis, MSD Oncology, Pierre Fabre, Roche/Genentech; Honoraria: Bristol Myers Squibb, MSD Oncology, Novartis, Pierre Fabre, Roche/Genentech; Research Funding: Novartis, Roche/Genentech; JL: Grants: Achilles Therapeutics, Aveo, Bristol Myers Squibb, MSD, Nektar, Novartis, Pierre Fabre, Pfizer, Pharmacyclics, Roche/ Genentech, Secarna, Vitaccess, Covance, Immunocore; Personal Fees: Achilles Therapeutics, AstraZeneca, Boston Biomedical, Bristol Myers Squibb, Eisai, EUSA Pharma, Imugen, Incyte, Ipsen, iOnctura, Kymab, Merck Serono, MSD, Nektar, Novartis, Pierre Fabre, Pfizer, Pharmacyclics, Roche/Genentech, Secarna, Vitaccess; PAA: Grant: Array, Bristol Myers Squibb, Roche/Genentech; Personal Fees: 4SC, Array, AstraZeneca, Bristol Myers Squibb, Idera, Immunocore, Incyte, Genmab, Medimmune, MSD, NewLink Genetics, Novartis, Merck Serono, Pierre Fabre, Roche/Genentech, Sandoz, Sanofi, Syndax, Sun Pharma, Ultimovacs; Advisory/ consultant role and travel support: MSD; MDV: Consultant/Advisory Boards: Bristol Myers Squibb, Merck, Novartis, Pierre Fabre, Sanofi; HG: Consulting or Advisory Role: Amgen, Bristol Myers Squibb, MSD Oncology, Novartis, Roche, Pierre Fabre; Travel, Accommodations, Expenses: Bristol Myers Squibb, Roche; Honoraria: Amgen, Bristol Myers Squibb, MSD Oncology, Novartis, Roche; Research Funding: Bristol Myers Squibb, MSD Oncology, Roche, Novartis; CLC: Nothing to disclose; AA: Personal Fees: Bristol Myers Squibb, MSD, Novartis, Merck, Roche; Travel Grant: Bristol Myers Squibb, MSD, Novartis, Merck, Roche; SD: Consulting or Advisory Role: MSD; Research Funding: AstraZeneca, Bristol Myers Squibb, MSD, Roche; Travel, Accommodations, Expenses: Bristol Myers Squibb; Stock Ownership/ Employment: Sanofi Pasteur (immediate family member); MS Grant: AbbVie, Amgen, AstraZeneca, Bristol Myers Squibb, Eli Lilly, Gilead, Merck Serono, MSD, Mylan, Novartis, Pfizer, Regeneron, Roche, Samsung; J-JG: Personal Fees: Amgen, Bristol Myers Squibb, MSD, Novartis, Pierre Fabre, Roche, Sanofi, SunPharma; VC-S: Advisory Board: Incyte, Merck-Sorono, MSD; Meeting Expenses: Bristol Myers Squibb, Pierre Fabre; Speaker Fee: Novartis; IM: Grant: Amgen, BionCoTech, Bristol Myers Squibb, Incyte, MSD, Roche; Personal Fees: Amgen, BionCoTech, Bristol Myers Squibb, Incyte, MSD, Regeneron, Roche, Sanofi; Non-financial Support: BionCoTech, Bristol Myers Squibb, MSD, Roche; MOB: Consulting or Advisory Role: Adaptimmune, Bristol Myers Squibb, EMD Serono, GlaxoSmithKline, Genzyme, Immunocore, Immunovaccine, Merck, Novartis; Expert Testimony: Merck; Honoraria: Bristol Myers Squibb, Merck, Novartis, Roche; Research Funding: Takara Bio, Merck; AMDG: Consulting or Advisory Role: Bristol Myers Squibb, Incyte, MSD, Pierre Fabre; $\mathrm{JL}$ : Consulting or Advisory Role: Array BioPharm, Bristol Myers Squibb, Novartis; Speakers' Bureau: Array BioPharm, Novartis, Regeneron; Travel, Accommodations, and Expenses: Bristol Myers Squibb, Novartis, Pfizer; Research Funding: Amgen, AstraZeneca/Medlmmune, Bristol Myers Squibb, Elios Pharmaceutical, Genentech/ Roche, Incyte, Merck Seronon, Merck, Novartis, Pfizer, Viralytics; LDLC-M: Nothing to disclose; VA: Personal Fees: Bristol Myers Squibb, Merck Serono, MSD, Novartis, Pierre Fabre, Roche, Oncosec; PA: Nothing to disclose; AH: Nothing to disclose; LF: Grant: Bristol Myers Squibb, EMD Serono, Incyte, Kartos, Merck, Pfizer, Array; Consulting or Advisory Role: Elsevier/Nia Oncology, Hoosier Cancer Research Network; MM: Consulting or Advisory Role: AstraZeneca, Boehringer Ingelheim, Bristol Myers Squibb, EMD Serono, MSD, Novartis, Pfizer, Roche; Travel, Accommodations, and Expenses: AstraZeneca, Bristol Myers Squibb, MSD, Roche; NIK: Consulting or Advisory Role: Array BioPharma, AstraZeneca, Bristol Myers Squibb, EMD Serono, Immunocore, HUYA Bioscience International, Genentech, Merck, Regeneron; Research Funding: Amgen, HUYA Bioscience International, GlaxoSmithKline, Merck, Novartis, Regeneron; Stock/Other Ownership Interests: Amarin Corporation, Bellicum Pharmaceuticals, Mazor Robotics, TransEnterix; VdP: Employee: Bristol Myers Squibb; Stock Ownership: Bristol Myers Squibb; ML: Employee: Bristol Myers Squibb; Stock Ownership: Advaxis Immunotherapies, Bristol Myers Squibb; JW: Consulting or Advisory Role: AbbVie Inc, Altor Bioscience, Amgen, AstraZeneca, Bristol Myers Squibb, CytomX Therapeutics, Daiichi Sankyo, Eisai, Genentech, GlaxoSmithKline, Medivation, Merck, Nektar Therapeutics,
Novartis, Pieris Pharmaceuticals, Inc, Roche, SELLAS Life Sciences Group, Inc, WindMIL Therapeutics; Research Funding: Astellas Pharma, Bristol Myers Squibb Genentech, Incyte Corporation, Merck, Novartis, Roche; Travel, Accommodations, and Expenses: Amgen, AstraZeneca, Bristol Myers Squibb, Daiichi Sankyo, Genentech, GlaxoSmithKline, Merck, Novartis, Pieris Pharmaceuticals, Inc, Roche; Honoraria: AbbVie Inc, Altor Bioscience, Amgen, AstraZeneca, Bristol Myers Squibb, CytomX Therapeutics, Daiichi Sankyo, Eisai, Genentech, GlaxoSmithKline, Medivation, Merck, Nektar Therapeutics, Novartis, Pieris Pharmaceuticals, Inc, Roche, SELLAS Life Sciences Group, Inc, WindMIL Therapeutics; Stock/Ownership Interests: Biond; Patents: Named on a patent submitted by Moffitt Cancer Center for an ipilimumab biomarker; named on a patent submitted from Biodesix for a PD-1 antibody biomarker.

\section{Patient consent for publication Not required.}

Ethics approval This trial was approved by the institutional review board or ethics committee at each center and complied with Good Clinical Practice Guidelines, the Declaration of Helsinki, and local laws. All patients provided written informed consent before any protocol-related procedure or treatment.

Provenance and peer review Not commissioned; externally peer reviewed.

Data availability statement Data are available upon reasonable request. BMS policy on data sharing may be found at HYPERLINK "https://protecteu.mimecast. com/s/8tv9CyQYCWkmRytMnYgD?domain=bms.com" https://www.bms.com/ researchers-and-partners/independent-research/data-sharing-request-process. html.

Supplemental material This content has been supplied by the author(s). It has not been vetted by BMJ Publishing Group Limited (BMJ) and may not have been peer-reviewed. Any opinions or recommendations discussed are solely those of the author(s) and are not endorsed by BMJ. BMJ disclaims all liability and responsibility arising from any reliance placed on the content. Where the content includes any translated material, BMJ does not warrant the accuracy and reliability of the translations (including but not limited to local regulations, clinical guidelines, terminology, drug names and drug dosages), and is not responsible for any error and/or omissions arising from translation and adaptation or otherwise.

Open access This is an open access article distributed in accordance with the Creative Commons Attribution Non Commercial (CC BY-NC 4.0) license, which permits others to distribute, remix, adapt, build upon this work non-commercially, and license their derivative works on different terms, provided the original work is properly cited, appropriate credit is given, any changes made indicated, and the use is non-commercial. See http://creativecommons.org/licenses/by-nc/4.0/.

\section{ORCID iDs}

Mario Mandalá http://orcid.org/0000-0001-8846-8959

Paolo A Ascierto http://orcid.org/0000-0002-8322-475X

Helen Gogas http://orcid.org/0000-0002-0451-2885

Ivan Marquez-Rodas http://orcid.org/0000-0002-2476-668X

Jose Lutzky http://orcid.org/0000-0002-9503-2130

Jeffrey Weber http://orcid.org/0000-0002-4962-2957

\section{REFERENCES}

1 Leiter U, Buettner PG, Eigentler TK, et al. Hazard rates for recurrent and secondary cutaneous melanoma: an analysis of 33,384 patients in the German central malignant melanoma registry. $J$ Am Acad Dermatol 2012;66:37-45.

2 Romano E, Scordo M, Dusza SW, et al. Site and timing of first relapse in stage III melanoma patients: implications for follow-up guidelines. J Clin Oncol 2010;28:3042-7.

3 Meyers MO, Yeh JJ, Frank J, et al. Method of detection of initial recurrence of stage II/III cutaneous melanoma: analysis of the utility of follow-up staging. Ann Surg Oncol 2009;16:941-7.

4 Tas F, Erturk K. Recurrence behavior in early-stage cutaneous melanoma: pattern, timing, survival, and influencing factors. Melanoma Res 2017;27:134-9.

5 National Cancer Institute. Cancer STAT facts: melanoma of the skin. Available: https://seer.cancer.gov/statfacts/html/melan.html [Accessed 30 Apr 2019].

6 Gershenwald JE, Scolyer RA, Hess KR, et al. Melanoma staging: evidence-based changes in the American joint Committee on cancer eighth edition cancer staging manual. CA Cancer J Clin 2017;67:472-92.

7 Eggermont AMM, Chiarion-Sileni V, Grob J-J, et al. Adjuvant ipilimumab versus placebo after complete resection of stage III 
melanoma: long-term follow-up results of the European organisation for research and treatment of cancer 18071 double-blind phase 3 randomised trial. Eur J Cancer 2019;119:1-10.

8 Tarhini AA, Lee SJ, Hodi FS, et al. Phase III study of adjuvant ipilimumab (3 or $10 \mathrm{mg} / \mathrm{kg}$ ) versus high-dose interferon alfa-2b for resected high-risk melanoma: North American intergroup E1609. J Clin Oncol 2020;38:567-75.

9 Coit DG, Thompson JA, Albertini MR, et al. Cutaneous melanoma, version 2.2019, NCCN clinical practice guidelines in oncology. J Nat/ Compr Canc Netw 2019;17:367-402.

10 Michielin O, van Akkooi ACJ, Ascierto PA, et al. Cutaneous melanoma: ESMO clinical practice guidelines for diagnosis, treatment and follow-upt. Ann Oncol 2019;30:1884-901.

11 Weber J, Mandala M, Del Vecchio M, et al. Adjuvant nivolumab versus ipilimumab in resected stage III or IV melanoma. $N$ Engl J Med 2017;377:1824-35.

12 Ascierto PA, Del Vecchio M, Mandalá M, et al. Adjuvant nivolumab versus ipilimumab in resected stage IIIB-C and stage IV melanoma (CheckMate 238): 4-year results from a multicentre, doubleblind, randomised, controlled, phase 3 trial. Lancet Oncol 2020;21:1465-77.

13 Balch CM, Gershenwald JE, Soong S-J, et al. Final version of 2009 AJCC melanoma staging and classification. J Clin Oncol 2009;27:6199-206.

14 National Cancer Institute. Cancer therapy evaluation program. common terminology criteria for adverse events (CTCAE).

Available: https://ctep.cancer.gov/protocolDevelopment/electronic_ applications/ctc.htm\#ctc_40 [Accessed 2 Jan 2020]
15 Giobbie-Hurder A, Gelber RD, Regan MM. Challenges of guaranteetime bias. J Clin Oncol 2013;31:2963-9.

16 Weber JS, Hodi FS, Wolchok JD, et al. Safety profile of nivolumab monotherapy: a pooled analysis of patients with advanced melanoma. J Clin Oncol 2017;35:785-92.

17 Teufel A, Zhan T, Härtel N, et al. Management of immune related adverse events induced by immune checkpoint inhibition. Cancer Lett 2019;456:80-7.

18 Freeman-Keller M, Kim Y, Cronin H, et al. Nivolumab in resected and unresectable metastatic melanoma: characteristics of immunerelated adverse events and association with outcomes. Clin Cancer Res 2016;22:886-94.

19 Hua C, Boussemart L, Mateus C, et al. Association of vitiligo with tumor response in patients with metastatic melanoma treated with pembrolizumab. JAMA Dermatol 2016;152:45-51.

20 Grimaldi A, Simeone E, Festino L, et al. Correlation between immunerelated adverse events and response to pembrolizumab in advanced melanoma patients. J Immunother Cancer 2015;3:P186.

21 Lutzky J, Wolchok J, Hamid O, et al. Association between immunerelated adverse events (irAEs) and disease control or overal survival in patients (PTS) with advanced melanoma treated with $10 \mathrm{mg} / \mathrm{kg}$ ipilimumab in three phase II clinical trials. JCO 2009;27:9034.

22 Eggermont AMM, Kicinski M, Blank CU, et al. Association between immune-related adverse events and recurrence-free survival among patients with stage III melanoma randomized to receive pembrolizumab or placebo: a secondary analysis of a randomized clinical trial. JAMA Oncol 2020;6:519-17. 